\title{
Electron cooling of a bunched ion beam in a storage ring
}

\author{
He Zhao, ${ }^{1,2}$ Lijun Mao, ${ }^{1, *}$ Jiancheng Yang, ${ }^{1}$ Jiawen Xia, ${ }^{1}$ Xiaodong Yang, ${ }^{1} \mathrm{Jie} \mathrm{Li},{ }^{1}$ \\ Meitang Tang, ${ }^{1}$ Guodong Shen, ${ }^{1}$ Xiaoming Ma, ${ }^{1}$ Bo Wu, ${ }^{1,2}$ Geng Wang, ${ }^{1,2}$ \\ Shuang Ruan, ${ }^{, 2}$ Kedong Wang, ${ }_{1,2}$ and Ziqiang Dong, \\ ${ }^{1}$ Institute of Modern Physics, Chinese Academy of Sciences, Lanzhou 730000, China \\ ${ }^{2}$ University of Chinese Academy of Sciences, Beijing 100049, China
}

(Received 20 January 2017; published 2 February 2018)

\begin{abstract}
A combination of electron cooling and rf system is an effective method to compress the beam bunch length in storage rings. A simulation code based on multiparticle tracking was developed to calculate the bunched ion beam cooling process, in which the electron cooling, intrabeam scattering (IBS), ion beam space-charge field, transverse and synchrotron motion are considered. Meanwhile, bunched ion beam cooling experiments have been carried out in the main cooling storage ring (CSRm) of the Heavy Ion Research Facility in Lanzhou, to investigate the minimum bunch length obtained by the cooling method, and study the dependence of the minimum bunch length on beam and machine parameters. The experiments show comparable results to those from simulation. Based on these simulations and experiments, we established an analytical model to describe the limitation of the bunch length of the cooled ion beam. It is observed that the IBS effect is dominant for low intensity beams, and the spacecharge effect is much more important for high intensity beams. Moreover, the particles will not be bunched for much higher intensity beam. The experimental results in CSRm show a good agreement with the analytical model in the IBS dominated regime. The simulation work offers us comparable results to those from the analytical model both in IBS dominated and space-charge dominated regimes.
\end{abstract}

DOI: 10.1103/PhysRevAccelBeams.21.023501

\section{INTRODUCTION}

The Heavy Ion Research Facility in Lanzhou-Cooling Storage Ring (HIRFL-CSR) accelerator complex is a multipurpose research facility for nuclear physics, atomic physics and heavy ion applied researches in medicine, biology and materials science [1]. It consists of two cyclotrons, two storage rings and several experimental terminals. The cyclotrons sector focusing cyclotron (SFC) and separated sector cyclotron (SSC) are used as the injector system. The main cooling storage ring (CSRm) is used for beam accumulation and acceleration. The experimental cooling storage ring (CSRe) provides a platform for nuclear and atomic experiments with heavy ions and rare isotopes. Two electron coolers installed in CSRm and CSRe are applied to the stored ion beams for phase space compression. The electron coolers were designed and manufactured in the cooperation between IMP China and BINP Russia [2]. Several distinctive technologies, such

\footnotetext{
*Corresponding author. maolijun@impcas.ac.cn

Published by the American Physical Society under the terms of the Creative Commons Attribution 4.0 International license. Further distribution of this work must maintain attribution to the author(s) and the published article's title, journal citation, and DOI.
}

as variable electron beam profile gun, pancake solenoid cooling section and electrostatic bending plate were used in these coolers firstly [3,4]. The layout of the HIRFL-CSR accelerator complex is shown in Fig. 1 and the detailed description can be found in Refs. [1,5,6].

Electron cooling is a powerful method to shrink the size, the divergence and the momentum spread of the stored charged-particle beams in storage rings for precision

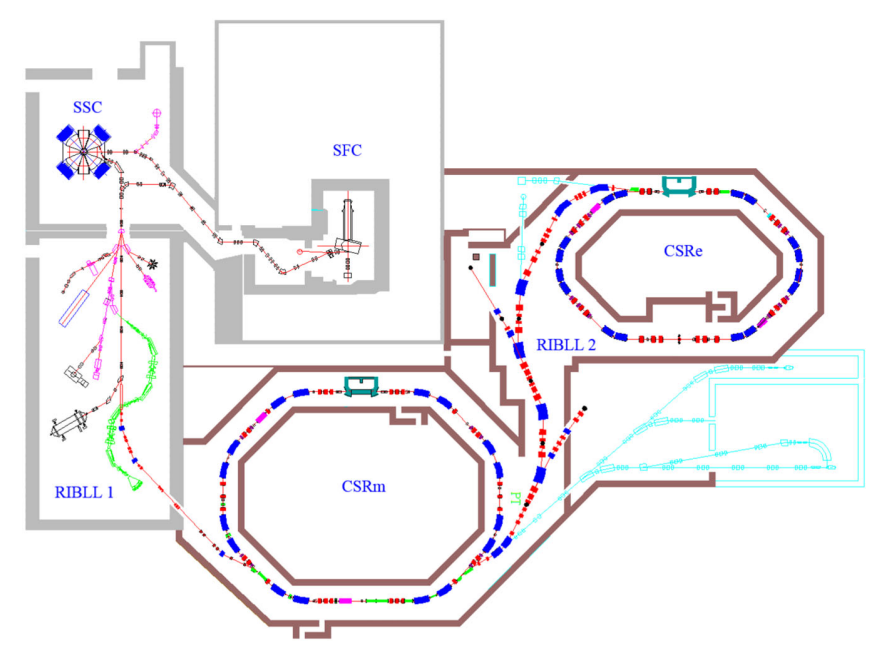

FIG. 1. Layout of the HIRFL-CSR accelerator complex. 
experiments [7]. It also supports beam manipulations involving the rf system to provide beams with short bunch length. Short-bunched ion beam has a wide range of applications in rare isotope production, high energy density physics experiments, collider and cancer therapy [8-10]. In order to study the cooling process of the bunched ion beam, a simulation code was developed, in which the electron cooling, intrabeam scattering (IBS) and space-charge effect are considered. Experimental investigations on the minimum bunch length obtained by the combination of electron cooling and a sinusoidal wave rf field were also carried out in CSRm under various intensities of cooled ${ }^{112} \mathrm{Sn}^{36+}$ and ${ }^{12} \mathrm{C}^{6+}$ ion beams.

CSRm is a racetrack shape synchrotron with the circumference of $161.0 \mathrm{~m}$. An electron cooler and a rf cavity are placed at the dispersion-free sections. The electron cooler with an effective length of $3.4 \mathrm{~m}$ works at the injection energies for beam accumulation. The rf cavity operates in the frequency range of 0.25 to $1.7 \mathrm{MHz}$ and the maximum voltage is $8.0 \mathrm{kV}$ [11]. Because of the low-frequency limitation of the rf cavity, the second harmonic is used at the injection energies, which means two bunches are produced in CSRm.

The bunched ion beam cooling process has been studied in several cooling storage rings, such as HIMAC, ESR, IUCF and CSRe [8,12-14]. The results show that the minimum bunch length of the cooled beam is determined by the equilibrium between electron cooling, IBS effect, ion beam space-charge field and rf voltage, but the dependence of the minimum bunch length on the beam parameters has a slight difference in those experiments. The purpose of this paper is to present the bunched ion beam cooling experiments in CSRm and introduce the bunched beam cooling simulation work based on the multiparticle tracking method. The experimental and simulated results are described and compared, according to which an analytical model is established to investigate the effects of IBS and space-charge on bunch length. Both the experimental and simulated results show a good agreement with the analytical model.

\section{SIMULATION OF BUNCHED BEAM COOLING}

The simulation code is developed based on multiparticle tracking, in which the ion beam is represented by a number of model particles and the beam dynamics is calculated by the statistical method. In the simulation procedure, the ring is divided into two parts. The first part is the cooling section and the second one is the rest of the ring. Before the model particles going into the cooling section, the beam dynamics is calculated on the basis of betatron and synchrotron oscillations (with rf voltage). Then the beam emittance and momentum spread will be cooled down when passing through the first part, and the bunch length will also be compressed. In order to obtain a more accurate result, the
IBS and longitudinal space-charge effect are carefully calculated for each turn.

In the code, a certain number of charged particles are generated based on the initial beam emittance, momentum spread and bunch length. Particularly, it assumes that the initial ion beam distribution is Gaussian in transverse and longitudinal. Each particle is presented as a six-coordinate vector: $\left(\mathrm{x}, \mathrm{x}^{\prime}, \mathrm{z}, \mathrm{z}^{\prime}, \phi, \Delta \mathrm{v}\right)$, where $\mathrm{x}$ and $\mathrm{z}$ are the horizontal and vertical coordinates, $\mathrm{x}^{\prime}$ and $\mathrm{z}^{\prime}$ are the corresponding angles in horizontal and vertical, $\phi$ is the phase angle with respect to the ring and $\Delta \mathrm{v}$ is the relative velocity of the particle in laboratory reference frame (LRF). Each coordinate is generated from Gaussian random value and the Twiss parameters. For each turn, the coordinate of each model particle will be tracked and the beam dynamics will be calculated according to the particles distribution. The details of the simulation code are described in Appendix A.

The calculation of electron cooling is based upon the energy exchange between ions and electrons, which can be described by a velocity-dependent friction force. In the simulation, the Parkhomchuk force formula is used at the particle reference frame (PRF) [15], and the electron density is around $3.8 \times 10^{6} \mathrm{~cm}^{-3}$ with uniform transverse distribution. In the cooling process, the friction force on each model particle is calculated by the relative velocity component between the ion and electron and the details can be found in Appendix B. Additionally, because of the space-charge potential of electron beam, the longitudinal velocities of the electrons at a certain radius $r$ should be corrected by [16]

$$
\frac{\Delta V_{e}}{V_{e}}=\frac{I_{e}}{4 \pi \varepsilon_{0} \beta^{3} \gamma^{3} c} \frac{e}{m_{e} c^{2}} \frac{r^{2}}{r_{b}^{2}},
$$

where $\varepsilon_{0}$ is the vacuum permittivity, $I_{e}$ is the electron beam current, $\beta$ and $\gamma$ are Lorentz factors, $c$ is the speed of light and $r_{b}$ is the radius of electron beam. As the particles pass through the cooling section, the emittance, momentum spread and bunch length of the ion beam will be rapidly compressed by the electron beam.

On the other hand, some heating effects which can induce beam blowups should be analyzed seriously, such as the IBS effect, target scattering and residual gas scattering [7]. In the code, these heating effects are all considered. Actually, only the IBS effect is important, because there is no internal target in CSRm and the vacuum pressure is very high. The IBS effect is a multiple Coulomb interaction of the charged particles within the beam. In the calculation, the Martini IBS model is applied, in which the growth rate is calculated from a complicated integration that connects the six-dimensional phase space density of the beam with the optics of the storage ring [17]. However, the growth rate $\lambda$ is described as the relative time derivatives of rms relative momentum spread and rms betatron angles, see Appendix C. 
TABLE I. Initial parameters used in simulation.

\begin{tabular}{lc}
\hline \hline Ion & ${ }^{12} \mathrm{C}^{6+}$ \\
Ion energy & $6.9 \mathrm{MeV} / \mathrm{u}$ \\
Particle number per bunch & $1.0 \times 10^{8}$ \\
Initial emittance (rms) & $2.0 / 1.0 \pi \mathrm{mm} \mathrm{mrad}$ \\
Initial momentum spread (rms) & $5.0 \times 10^{-4}$ \\
Initial beam length (rms) & $3.8 \mathrm{~m}$ \\
Ring circumference & $161.0 \mathrm{~m}$ \\
Length of the cooler & $3.4 \mathrm{~m}$ \\
Betatron tune & $3.63 / 2.61$ \\
$\beta$ value in the cooling section & $10 / 10 \mathrm{~m}$ \\
$\alpha$ value in the cooling section & $0.0 / 0.0$ \\
Dispersion in the cooling section & $0.0 \mathrm{~m}$ \\
Transition gamma $\gamma_{\mathrm{tr}}$ & 5.42 \\
rf voltage & $1.0 \mathrm{kV}$ \\
Harmonic number & 2.0 \\
Electron beam density & $3.8 \times 10^{6} \mathrm{~cm}{ }^{-3}$ \\
Electron beam diameter & $50 \mathrm{~mm}$ \\
Transverse e-beam temperature & $0.5 \mathrm{eV}$ \\
Longitudinal e-beam temperature & $1.0 \times 10^{-5} \mathrm{eV}$ \\
Magnetic field in the cooling section & $365.0 \mathrm{Gs}$ \\
\hline \hline
\end{tabular}

The ion beam density increases with the cooling process, during which the space-charge effect becomes much stronger to prevent the cooling effect on bunch length and beam profile accordingly. In the code, the space-charge effect is considered only in the longitudinal and this effect is represented by a potential that is similar to the rf voltage. The space-charge potential is given by [18]

$$
V_{\mathrm{SC}}=\frac{g h^{2}}{2 R \varepsilon_{0} \gamma^{2}} \frac{d \rho(\phi)}{d \phi},
$$

in which $\rho$ is the linear charge density of ion beam, $h$ is the harmonic number and $R$ is the radius of the ring. The geometric factor $g=1+2 \ln \frac{b}{a}$ depends on the ratio of beam radius $a$ to pipe radius $b$. The change of particle velocity caused by the space-charge potential together with the rf voltage for each turn is calculated, which is based on the synchrotron motion and more details can be found in Appendix A.

An application is dedicated to the bunched beam cooling for the typical ion beam ${ }^{12} \mathrm{C}^{6+}$ at the injection energy of 6.9 MeV/u on CSRm. The main parameters used in the simulation are listed in Table I. The variations of the beam emittance, momentum spread and bunch length with time under the combined actions of the IBS, space-charge and electron cooling are shown in Fig. 2. The distribution of beam momentum spread and beam bunch length in the cooling process are shown in Fig. 2 (contour map) to reveal the evolution of particle distribution. Initially, both the momentum spread and the bunch length decrease quickly since the cooling effect is much stronger than other heating effects. Afterward, the IBS and space-charge effect become stronger due to the increased density of the beam during the
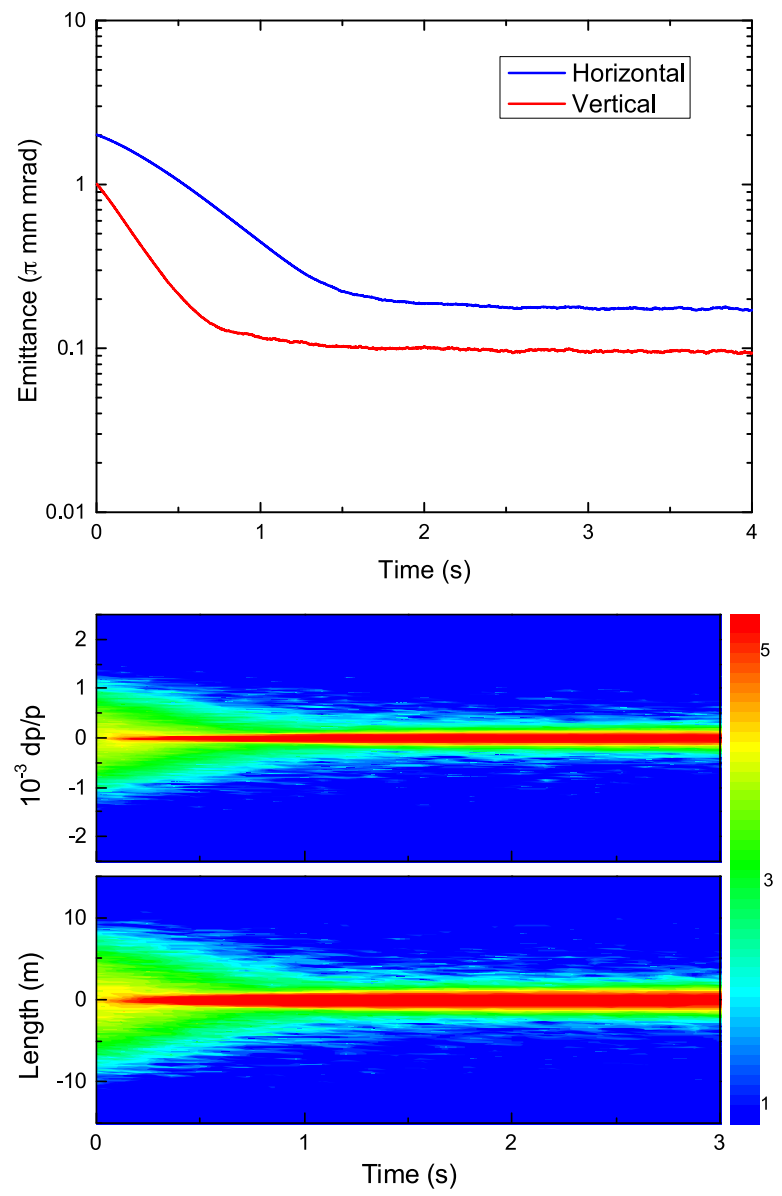

FIG. 2. Evolution of beam emittance (rms), distribution of momentum spread and bunch length in the procedure of cooling combined with rf field $\left(V_{r f}=1.0 \mathrm{kV}\right)$ for $6.9 \mathrm{MeV} / u^{12} \mathrm{C}^{6+}$ beam.

cooling process. Finally, the balance of cooling and heating is reached after about 2 seconds.

The particle distribution in longitudinal phase space, the corresponding separatrix orbit and the beam space charge potential during the cooling process are given in Fig. 3. The space-charge potential shows the behavior increasing with the decrease of bunch length as it was cooling down, during which the bunch length is prevented from being cooled to much shorter together with the IBS effect. However, the space-charge potential at 1 second is larger than that at the equilibrium state and many particles are cooled to the center as shown in Fig. 3. It is mainly because the cooling force in the longitudinal is larger than that in the transverse. Many particles are quickly cooled to the center in the longitudinal at the beginning of cooling and then diffuse slowly until the equilibrium state is achieved. For a cooled ion beam with stationary distribution, the particle charge density in the longitudinal can be described by the FokkerPlank equation [19],

$$
\rho(s) e^{-\alpha \rho(s)}=\rho(0) e^{-\alpha \rho(0)} e^{-s^{2} / 2 \sigma_{b}^{2}},
$$




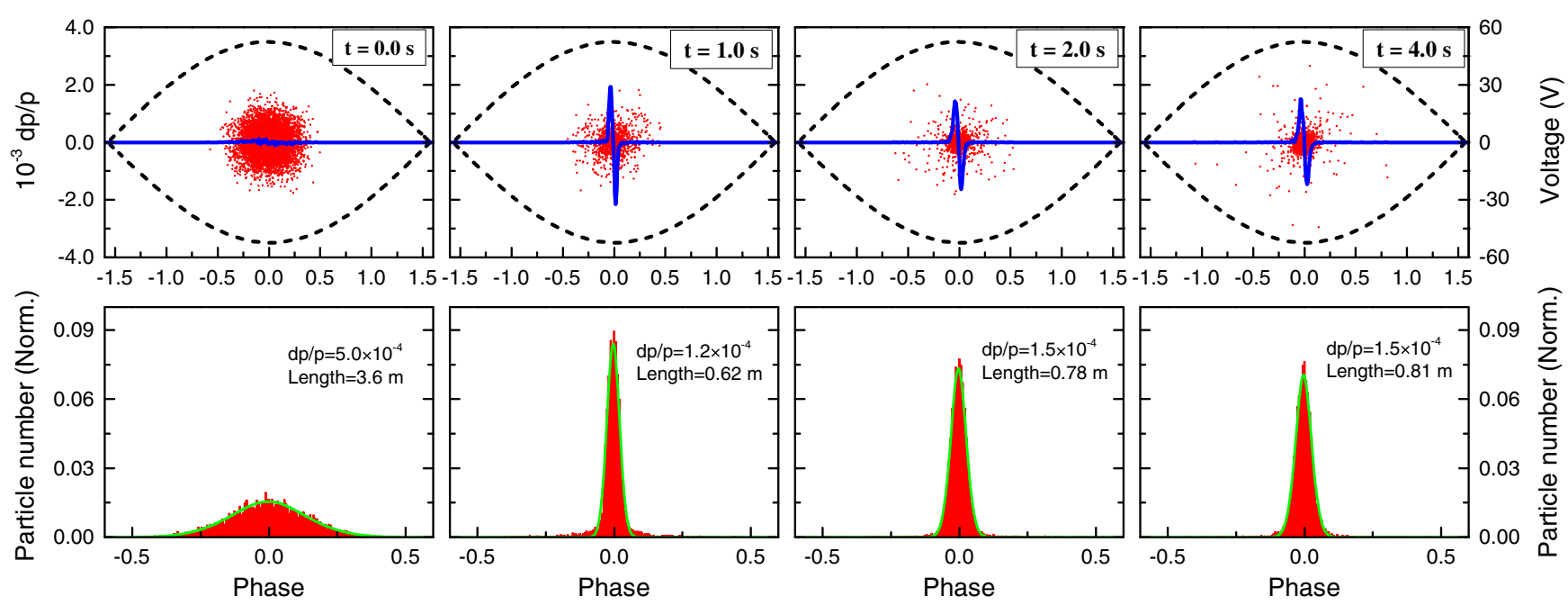

FIG. 3. Evolution of the model particles distribution in longitudinal phase space (red dot), the space-charge potential (blue line) and the particles distribution in the longitudinal for one bunch of ${ }^{12} \mathrm{C}^{6+}$ beam in CSRm. The black dashed line is the rf bucket with $V_{r f}=1.0 \mathrm{kV}$ and the green line is the fitting result by the Fokker-Plank equation. The fitting result of bunch length and momentum spread is given.

where $\rho(0)$ is used to normalize the total charge of the beam. The constants $\alpha$ and $\sigma_{b}$ are given by the equations,

$$
\begin{gathered}
\alpha=\frac{Z_{0} g}{\gamma^{2} \beta} \frac{e f_{0}}{E \beta^{2}} \frac{R}{\sigma^{2} \eta} \\
\sigma_{b}=\left(\frac{2 \pi \beta^{2}|\eta|}{h} \frac{E}{e V_{r f}}\right)^{1 / 2} R \sigma,
\end{gathered}
$$

where $g$ is the geometrical factor and $\sigma$ represents the longitudinal beam temperature. This equation is used to fit the beam distribution during cooling and the fitting results have a good agreement with the simulation results as shown in Fig. 3.

As mentioned above, the minimum bunch length of the cooled beam is determined by the equilibrium between electron cooling, IBS effect and space-charge effect. In order to verify the simulation results, experiments were carried out in CSRm. The minimum bunch length reduced by the combination of electron cooling and rf field is measured.

\section{EXPERIMENT OF THE BUNCHED BEAM COOLING IN CSRM}

\section{A. Bunch length measurement}

The experiments were performed with ${ }^{112} \mathrm{Sn}^{36+}$ beam at the energy of $3.7 \mathrm{MeV} / \mathrm{u}$ and ${ }^{12} \mathrm{C}^{6+}$ beam at the energy of $6.9 \mathrm{MeV} / \mathrm{u}$, respectively. The range of the stored particle number was from $10^{6}$ to $10^{8}$. A flat distribution electron beam with the diameter around $50 \mathrm{~mm}$ was used for beam cooling. The electron beam current was set as $135 \mathrm{~mA}$ for ${ }^{112} \mathrm{Sn}^{36+}$ beam and $44 \mathrm{~mA}$ for ${ }^{12} \mathrm{C}^{6+}$ beam, respectively. A sinusoidal rf voltage from 0.2 to $2.3 \mathrm{kV}$ was applied with the harmonic number of 2 . The electron cooling and $\mathrm{rf}$ system parameters in the experiments are summarized in Table II. The same lattice parameters were used for ${ }^{112} \mathrm{Sn}^{36+}$ and ${ }^{12} \mathrm{C}^{6+}$ experiments, which were listed in Table I.

A typical experimental cycle is as follows: beams are injected, accumulated and cooled with the help of the continuous electron beam, and then a sinusoidal rf voltage is switched on. The bunch length after 2 seconds of turning on the rf system is measured by a position pickup with the length of $150 \mathrm{~mm}$ and the capacitor of $120 \mathrm{pF}$. The voltage drop at the 50 Ohms resistor between the pickup and ground is amplified by a preamplifier with the gain factor of $54 \mathrm{~dB}$. An oscilloscope with the bandwidth of $1 \mathrm{GHz}$ is used to read the signal from the preamplifier. The rms bunch length is evaluated from the Fokker-Plank fitting result of the voltage signal. As an example, a measurement of the voltage signal (black line) from Beam Position Monitor (BPM) for ${ }^{112} \mathrm{Sn}^{36+}$ beam is shown in Fig. 4. In this case, the beam longitudinal linear charge density is obtained by the integration of the pickup voltage signal (red line). The rms

TABLE II. Electron cooling and rf system parameters in the experiments.

\begin{tabular}{lcc}
\hline \hline Ion & ${ }^{112} \mathrm{Sn}^{36+}$ & ${ }^{12} \mathrm{C}^{6+}$ \\
Ion energy $[\mathrm{MeV} / \mathrm{u}]$ & 3.7 & 6.9 \\
Particle number per bunch $\left[\times 10^{7}\right]$ & $0.2-3$ & $2.5-20$ \\
rf voltage $[\mathrm{kV}]$ & $0.2-1.4$ & $0.3-2.3$ \\
Harmonic number & 2 & 2 \\
Electron energy [keV] & 2.0 & 3.7 \\
Electron beam current $[\mathrm{mA}]$ & $\sim 135$ & $\sim 44$ \\
Electron beam diameter [mm] & $\sim 50$ & $\sim 50$ \\
Magnetic field in cooling section [Gs] & $\sim 365$ & $\sim 365$ \\
\hline \hline
\end{tabular}




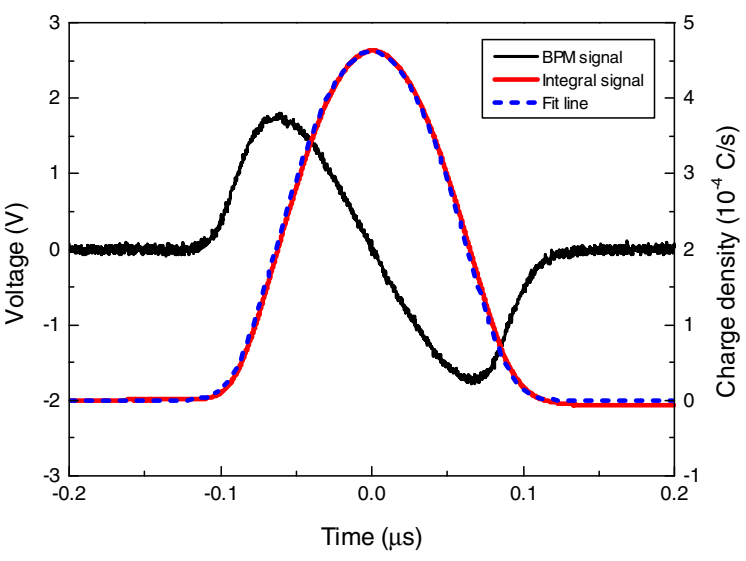

FIG. 4. The measured BPM signal (black) of the bunched beam, the beam linear charge density (red) calculated from the BPM signal and the Fokker-Plank fitting result (blue) in the experiment of $3.7 \mathrm{MeV} / \mathrm{u}^{112} \mathrm{Sn}^{36+}$. The rms bunch length is about $28 \pm 0.2 \mathrm{~ns}\left(I=17.7 \mu \mathrm{A}, V_{r f}=1.4 \mathrm{kV}, g=3.7, \sigma=\right.$ $\left.2.3 \times 10^{-4}\right)$.

bunch length $\sigma_{t}$ is evaluated by Fokker-Plank fitting of the bunch shape, as the blue line shown in Fig. 4. In the fitting process, the geometric factor $g$ and the momentum spread $d p / p$ are the two main factors to determine the bunch length and the particle distribution.

The rms bunch length $\sigma_{b}$ is obtained via

$$
\sigma_{b}=\sigma_{t} f C,
$$

where $f$ is the revolution frequency measured by the Schottky detector and $C$ is the circumference of CSRm. An estimate of the errors in the bunch length measurement is summarized in Table III. The uncertainty for the calculation of $\sigma_{b}$ is dominated by the measured revolution frequency, the intrinsic response of pickup and the fitting result. A relative error of the bunch length in the measurement is about $\pm 6 \%$, in which the intrinsic response error of the pickup is the main factor.

The stored beam current was measured by the dc current transformer (DCCT) in CSRm. The particle number per bunch is calculated by

$$
N=\frac{I}{e Z h f},
$$

TABLE III. Estimate errors for the bunch length measurement.

\begin{tabular}{lcc}
\hline \hline Name & Error (\%) & \multicolumn{1}{c}{ Source } \\
\hline Revolution frequency & \pm 1 & Schottky detector \\
Measured data & \pm 5 & Response of pickup \\
Data analysis & \pm 0.5 & $\begin{array}{c}\text { Standard deviation of } \\
\text { the measured data }\end{array}$ \\
$\begin{array}{l}\text { Particle number } \\
\text { rf amplitude }\end{array}$ & \pm 4 & DCCT measurement \\
\hline \hline
\end{tabular}

where $I$ is the beam current measured by DCCT, $Z$ is the charge state of ions and $h$ is the harmonic number. The uncertainty in determining $N$ is dominated by the accuracy of the current measurement, which is about $\pm 4 \%$. Additionally, the uncertainty of the rf voltage applied on the ion beam is dominated by the rf device output. All of these errors are summarized in Table III.

\section{B. Experimental results and analysis}

The experimental results of ${ }^{112} \mathrm{Sn}^{36+}$ and ${ }^{12} \mathrm{C}^{6+}$ beam are shown in Fig. 5, in which the dependencies of the minimum bunch length on particle number for the different rf voltages are presented. It is observed that the bunch length increases proportionally to $N^{k}$, and the range of $k$ is from 0.22 to 0.28 for such low ion intensities and energies. For spacecharge dominated beam, $k$ is close to $1 / 3$, which has been measured and analyzed in Refs. [8,14]. According to Ref. [19], the beam charge density in the longitudinal is determined by the geometrical factor $g$ and $\sigma$ that $g$ influences the width of the central part and $\sigma$ defines the beam tail. In our case, we think the bunched beam after cooling belongs to the IBS domain and the bunch length is
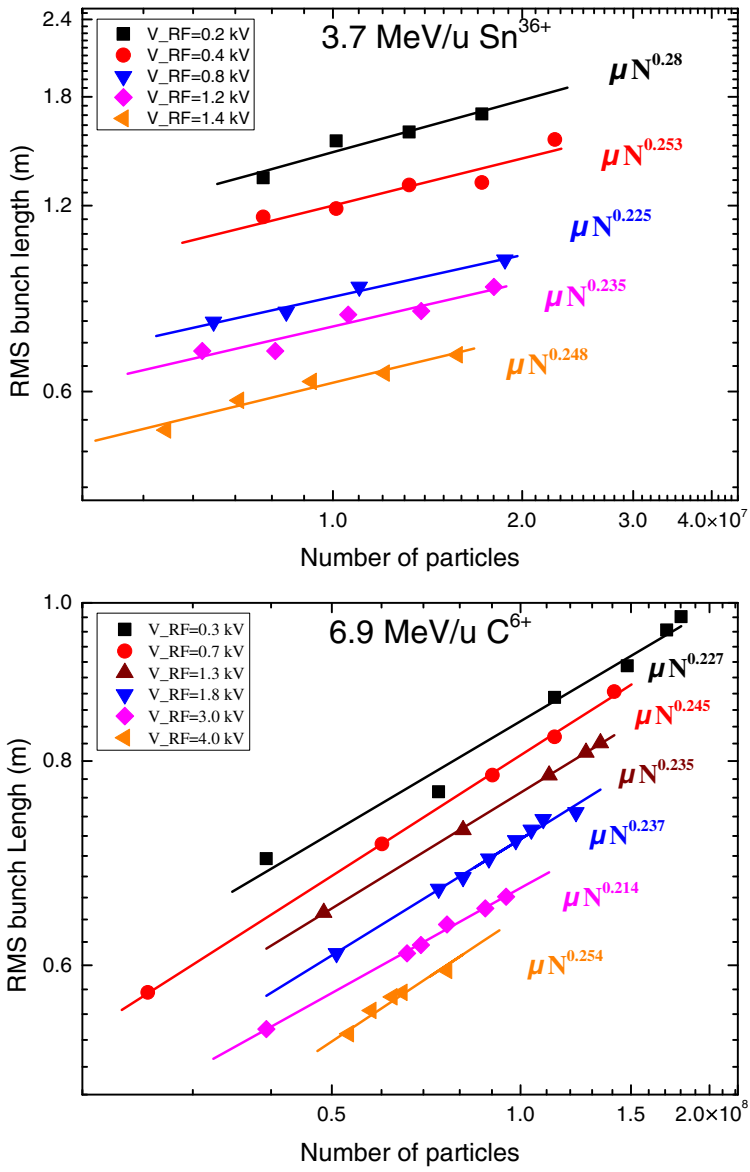

FIG. 5. Dependency of the rms bunch length on the stored particle number for different rf amplitude for $3.7 \mathrm{MeV} / \mathrm{u}$ ${ }^{112} \mathrm{Sn}^{36+}$ and $6.9 \mathrm{MeV} / \mathrm{u}{ }^{12} \mathrm{C}^{6+}$ ion beams. 
mainly determined by the IBS effect, even the central part of the beam is strongly affected by the space-charge effect. As the results shown in Ref. [19], the evolution of the rms momentum spread has different rules for the total beam current and the peak current, respectively.

In the experiments, the stored particle number per bunch is from $10^{6}$ to $10^{8}$. Accordingly, the IBS effect is the main heating source to affect the bunch length for such low intensity and low energy ion beams, in which the spacecharge effect only exists at the central part of the beam that with quite small momentum spread. Therefore, the distribution of the bunched beam is mainly determined by the particles outside the center that with large momentum spread relatively. The space-charge potential can be ignored for the particles outside the center, and the synchrotron motion of these particles can be described by the smallamplitude oscillation in the rf bucket [20],

$$
\frac{\hat{\delta}}{\hat{\theta}}=\left(\frac{e h V_{r f}\left|\cos \phi_{s}\right|}{2 \pi \beta^{2} E|\eta|}\right)^{1 / 2}=\frac{Q_{s 0}}{|\eta|},
$$

where $\hat{\delta}$ and $\hat{\theta}$ are the maximum amplitudes of the phase space ellipse, $E$ is the total energy of ions, $\eta$ is the slip factor and $Q_{s 0}$ is the synchrotron tune. It is clear that the bunch length for the small-amplitude oscillation increases linearly with the momentum spread for a certain rf amplitude. In the cooling process, the evolution of the beam momentum spread satisfies the differential equation

$$
\frac{1}{\delta_{p}} \frac{d \delta_{p}}{d t}=-\frac{1}{\tau_{\text {cooling }}}+\lambda_{\text {heating }}
$$

where $\tau_{\text {cooling }}$ is the cooling time and $\lambda_{\text {heating }}$ is the heating rate. For low ion intensities and energies, the heating rate is determined by the IBS heating rate $\lambda_{\text {IBS }}$. When the equilibrium is achieved, we get

$$
\frac{1}{\tau_{\text {cooling }}}=\lambda_{\mathrm{IBS}}
$$

According to the electron cooling theory, the cooling force on the particle with a small momentum deviation $\Delta p$ is a linear function $F=k \Delta p$, where $k$ is the slope of the longitudinal cooling force which is a constant for a certain electron beam setting [21]. So, the cooling time for the cooled beam at the equilibrium status is

$$
\frac{1}{\tau_{\text {cooling }}}=\frac{F}{p_{0}}=k \delta_{p}
$$

The gas relaxation model can be used for the calculation of the IBS heating rate [22]. The longitudinal heating rate for bunched beam is given by

$$
\lambda_{\mathrm{IBS}}=\frac{1}{2 \delta_{p}^{2}} \frac{d \delta_{p}^{2}}{d t}=\frac{r_{i}^{2} c N_{i} \Lambda}{16 \beta^{2} \gamma^{3} \varepsilon_{\perp}^{3 / 2}\left\langle\beta_{\perp}^{1 / 2}\right\rangle \sqrt{\pi / 2} \sigma_{b} \delta_{p}^{2}},
$$

where $\Lambda$ is the Coulomb logarithm, $\varepsilon_{\perp}$ is the transverse emittance, $\beta_{\perp}$ is the beta function, \langle\rangle is the average value of the ring lattice and $N_{i}$ is the particle number that corresponds to $92 \%$ of ion beam in the longitudinal. In the equilibrium state, we get

$$
k \delta_{p}=\frac{r_{i}^{2} c N_{i} \Lambda}{16 \beta^{2} \gamma^{3} \varepsilon_{\perp}^{3 / 2}\left\langle\beta_{\perp}^{1 / 2}\right\rangle \sqrt{\pi / 2} \sigma_{b} \delta_{p}^{2}} .
$$

According to Eqs. (8) and (13), the minimum bunch length after cooling is proportional to $N^{1 / 4}$,

$$
\sigma_{b} \propto N^{1 / 4}
$$

which has a good agreement with the experiment results shown in Fig. 5. Additionally, a series of experimental studies at ESR and IUCF $[12,13]$ have shown that the increase of the momentum spread $\delta_{p}$ with the number of stored particles $N$ for one bunch is evidenced as

$$
\delta_{p} \propto\left(\frac{N}{B_{f}}\right)^{1 / 3},
$$

where $B_{f}=I_{\text {average }} / I_{\text {peak }}$ is the bunch factor and $B_{f}=1$ for coasting beam. In the case of bunched beam in the IBS domain, the beam distribution in the longitudinal is much closer to Gaussian, for which the bunch factor can be achieved by $B_{f}=\sqrt{2 \pi} h \sigma_{b} / C$, where $C$ is the ring circumference. According to Eqs. (8) and (15), we can also get the bunch length is proportional to $N^{1 / 4}$. Furthermore, the minimum momentum spread is limited by the IBS heating, which is proportional to $Z^{4} / A^{2}$ [22]. It means that the bunch length of the ${ }^{112} \mathrm{Sn}^{36+}$ ion beam is usually larger than the ${ }^{12} \mathrm{C}^{6+}$ ion beam under similar conditions, which can be seen in Fig. 5. Therefore, we conclude that the experimental results in CSRm demonstrate that the IBS effect is dominant for such low intensity and low energy beams. But the space-charge effect will strongly affect the central distribution of the beam.

\section{BENCHMARK FOR THE SIMULATION CODE}

The experiments of the bunched beam cooling for ${ }^{112} \mathrm{Sn}^{36+}$ and ${ }^{12} \mathrm{C}^{6+}$ ions in CSRm were simulated by the cooling program. The dependencies of the final beam emittance and the bunch length on particle number are shown in Figs. 6 and 7. The simulation results have a good agreement with the measurements, as shown in Fig. 7, in which the rms bunch length is fitted by the Fokker-Plank equation at the equilibrium state. In the simulation, the electron density is $1.6 \times 10^{7} \mathrm{~cm}^{-3}$ and $3.8 \times 10^{6} \mathrm{~cm}^{-3}$ for 


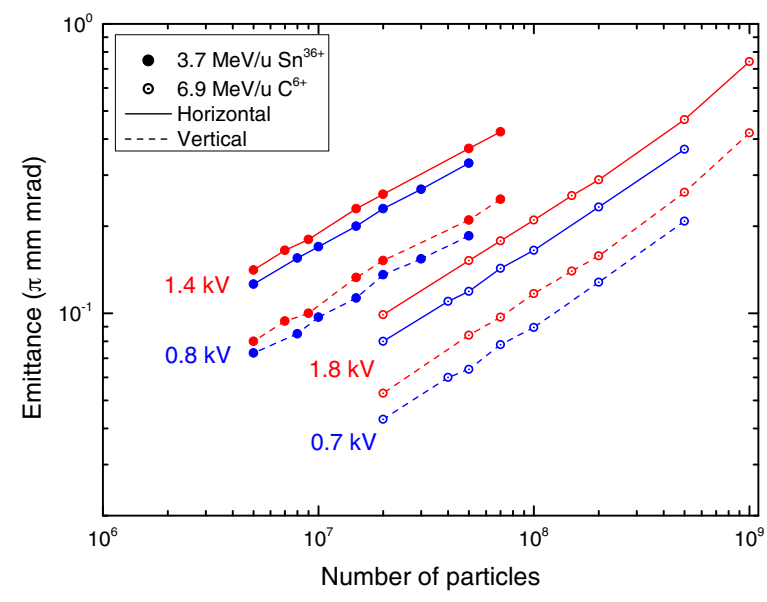

FIG. 6. The dependence of the beam emittance on particle number for the beam ${ }^{112} \mathrm{Sn}^{36+}$ and ${ }^{12} \mathrm{C}^{6+}$ in the simulation.
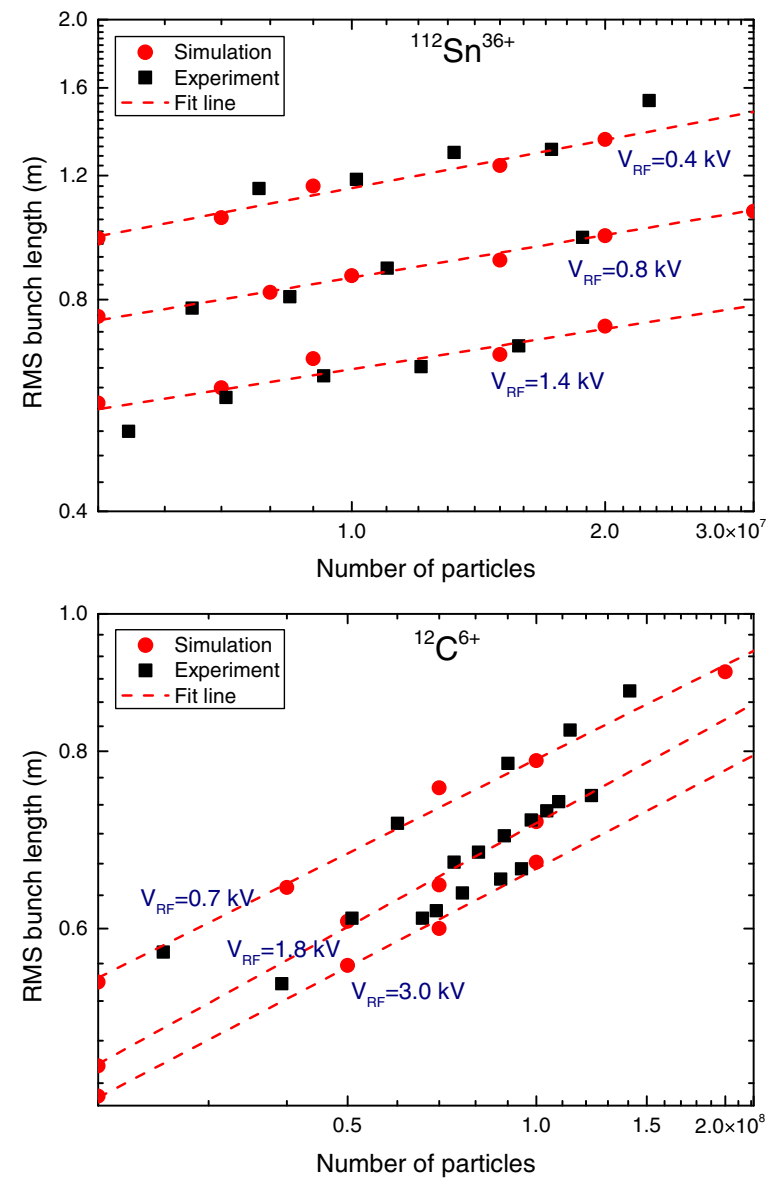

FIG. 7. Comparison of the bunch length between simulation and experiment. The dashed lines are fitted to the simulation results.

${ }^{112} \mathrm{Sn}^{36+}$ and ${ }^{12} \mathrm{C}^{6+}$ beams, respectively. The parameters used in the simulation are listed in Table IV.

In order to investigate the limitation of the bunch length for high intensity ion beam, more calculations on
TABLE IV. The parameters used in the simulation for benchmark.

\begin{tabular}{lcc}
\hline \hline Ion & ${ }^{112} \mathrm{Sn}^{36+}$ & ${ }^{12} \mathrm{C}^{6+}$ \\
Energy $[\mathrm{MeV} / \mathrm{u}]$ & 3.7 & 6.9 \\
Electron energy [keV] & 2.0 & 3.8 \\
Electron beam density $\left[\mathrm{cm}^{-3}\right]$ & $1.6 \times 10^{7}$ & $3.8 \times 10^{6}$ \\
Transverse temperature $[\mathrm{eV}]$ & 0.5 & 0.5 \\
Longitudinal temperature $[\mathrm{eV}]$ & $1.0 \times 10^{-5}$ & $1.0 \times 10^{-5}$ \\
\hline \hline
\end{tabular}

$6.9 \mathrm{MeV} / \mathrm{u}^{12} \mathrm{C}^{6+}$ beam with rf voltage $1.0 \mathrm{kV}$ were done. The particle number is from $10^{7}$ to $10^{10}$ per bunch. In the calculation, the IBS and space-charge effect are considered independently, and the results comparing with the measurements are shown in Fig. 8. According to the results considered the IBS effect only, the experiments of ${ }^{12} \mathrm{C}^{6+}$ in CSRm clearly belong to the IBS dominated regime. The bunch length is proportional to $\mathrm{N}^{1 / 4}$, which has a good agreement with Eq. (15). The space-charge dominated beam is obtained when the particle number per bunch exceeds $6 \times 10^{8}$. When only the space-charge effect is considered, it is observed that the dependence of the bunch length on particle number is divided into two regions. According to Ref. [14], the longitudinal phase oscillation is determined by the rf and space-charge voltage,

$$
\begin{gathered}
\Omega_{s}^{2}=\Omega_{s 0}^{2}\left(1-k \frac{N}{\sigma_{b}^{3}}\right), \\
k=\frac{1}{\gamma^{2}} \frac{Z e}{4 \pi \epsilon_{0}}\left[1+2 \ln \left(\frac{b}{a}\right)\right] \frac{\sqrt{2 \pi} R^{2}}{U_{0}},
\end{gathered}
$$

where $\Omega_{s 0}$ is the synchrotron angular frequency without any space-charge effect. It is a constant value for small-amplitude oscillation

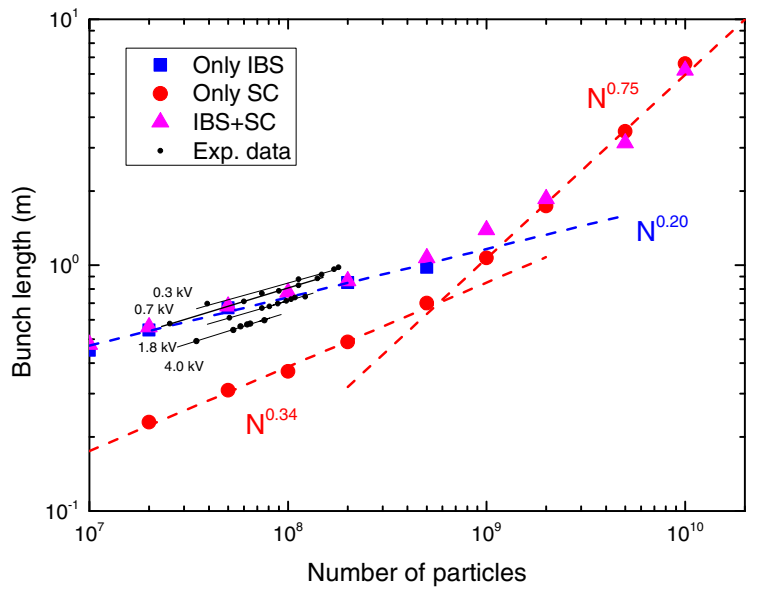

FIG. 8. The bunch length versus particle number in the simulation (colored) and experiment (black) for $6.9 \mathrm{MeV} / \mathrm{u}$ ${ }^{12} \mathrm{C}^{6+}$ ion beam. In the simulation, the bunch length is estimated by considering the effects of IBS and space charge independently. The dashed lines are fitted to the simulation results. 


$$
\Omega_{s 0}=\sqrt{\frac{\omega_{0}^{2} e h U_{r f}|\eta|}{2 \pi \beta^{2} E}},
$$

where $\omega_{0}$ is the angular revolution frequency of the reference particle and $E$ is the particle energy. By considering $\sigma_{b} \Omega_{s}=$ $\sigma_{b 0} \Omega_{s 0}$ and $\left(\sigma_{b 0} / \sigma_{b}\right)^{2} \ll 1, \sigma_{b}$ is approximately given by

$$
\sigma_{b} \doteq(k N)^{1 / 3} .
$$

The expected dependence of the bunch length shown in Fig. 8 is close to $N^{1 / 3}$ for the particle number less than $10^{9}$. The simulation result can be explained rather well by the equilibrium between the space-charge effect and the rf voltage.

Beyond this particle number, an additional increase of the bunch length occurs, proportional to $\mathrm{N}^{0.75}$. It is also observed that some particles are outside the rf bucket. In this case, we thought one of the reasons is the largeamplitude synchrotron caused by the strong space-charge effect. For synchrotron motion, the small-amplitude oscillation is deduced by

$$
\sin \phi-\sin \phi_{s} \doteq\left(\phi-\phi_{s}\right) \cos \phi_{s},
$$

where $\phi$ is the phase angle for a nonsynchronous particle. If the phase angle is large, the synchrotron motion will not satisfy Eq. (16) and the nonlinear effect will happen. We estimate that the critical phase angle of small-amplitude oscillation is $\pi / 6$, which means the critical bunch length $(\mathrm{rms})$ is about $0.8 \mathrm{~m}$ for our case that the ring circumference is $161 \mathrm{~m}$ and the harmonic number is 2 . It shows a good agreement with the simulation result shown in Fig. 8.

According to the simulation and experiments, we can give the estimate of the limitation of the bunch length that satisfies the theoretical analysis. The simulation code can almost apply to any kind of heavy ion beams with different energies, and can distinguish the IBS dominated beam or space-charge dominated beam.

\section{SUMMARY AND OUTLOOK}

In this paper, we reported the experimental and simulation results of the minimum ion beam bunch length obtained by a combination of electron cooling and rf system. It is obvious that such a combination is an effective method for beam bunch compression. Both results show that the minimum bunch length is proportional to $N^{\alpha}$. According to the theoretical analysis and particle tracking simulation, the dependence can be divided into two conditions. For small particle number per bunch and low energy like our case, ions move along the linearized synchrotron oscillation. The IBS effect is dominant and the exponent $\alpha=1 / 4$, but the space-charge effect does exist to affect the central part of beam. For some other cases with larger energy like in HIMAC and CSRe, the ions will be affected by the rf system and ion beam space-charge field. The ion beam space-charge effect is dominant and the exponent $\alpha=1 / 3$. Additionally, when more particles are applied, the blowup effect will happen and the beam almost cannot be bunched by the rf voltage. The experimental results in CSRm show a good agreement with the simulation results in the IBS dominated regime.

However, the simulation code lacks the calculation of the transverse space-charge effect, which may affect the longitudinal distribution of the bunched beam. So, the results of the bunch length are not perfect, especially for the high intensity ion beam. Further experiments and simulations for high energy and high intensity beam will be carried out in a next step to investigate the influence of the transverse spacecharge effect on bunch length. The simulation code will be also developed for further studies of bunched beam cooling, especially the study of pulse electron beam cooling, in preparation for which the cooling system of CSRm will be updated.

\section{ACKNOWLEDGMENTS}

The authors would like to express their thanks to members of the HIRFL-CSR operation group. We also thank Dr. Parkhomchuk for giving many suggestions on the experiment. We give special thanks to Dr. Katayama and Dr. Smirnov for the help on the simulation code. This work is supported by the National Natural Science Foundation of China (Projects No. 11575264, No. 11375245 and No. 11475235) and the Hundred Talents Project of the Chinese Academy of Sciences.

\section{APPENDIX A: DETAILS OF SIMULATION CODE}

The electron cooling simulation platform is a macroparticle simulation code built with FORTRAN. At the beginning of the code, the beam with Gaussian distribution will be generated according to initial rms beam emittance, momentum spread and bunch length,

$$
\begin{aligned}
& \left(x, x^{\prime}, z, z^{\prime}, \phi, \Delta v\right) \\
& =\xi\left(\sqrt{\varepsilon_{x} \beta_{x}}, \sqrt{\frac{\varepsilon_{x}}{\beta_{x}}}, \sqrt{\varepsilon_{z} \beta_{z}}, \sqrt{\frac{\varepsilon_{z}}{\beta_{z}}}, \delta_{b}, \delta_{p} V_{0}\right),
\end{aligned}
$$

where the random number with standard normal Gaussian distribution $\xi$ is encoded by the formula [23]

$$
\xi=\sqrt{-2 \operatorname{Ln}\left(\xi_{1}\right)} \sin \left(2 \pi \xi_{2}\right),
$$

where $\xi_{1}$ and $\xi_{2}$ are two uniform random numbers from zero to one. After this, each particle will be tracked turn by turn in the cooling process, during which the betatron and synchrotron motion are calculated. After each turn, the beam emittance, momentum spread and bunch length will be calculated based on statistical method. The IBS heating rate and the space-charge potential will also be calculated 
according to the new beam distribution and applied to the next turn.

\section{Betatron motion}

In the simulation code, the transmission of the particles in transverse is based on the betatron oscillation and the kick of electron cooling and IBS effect, which can be expressed by

$$
\begin{aligned}
\left(\begin{array}{l}
x_{j} \\
x_{j}^{\prime}
\end{array}\right)_{n+1}= & \left(\begin{array}{cc}
1 & 0 \\
0 & k_{j_{\text {cool }}}+k_{j_{\mathrm{IBS}}}
\end{array}\right)\left(\begin{array}{cc}
\cos \left(\Phi_{j}\right) & \beta_{j} \sin \Phi_{j} \\
-\gamma_{j} \sin \Phi_{j} & \cos \Phi_{j}
\end{array}\right) \\
& \times\left(\begin{array}{c}
x_{j} \\
x_{j}^{\prime}
\end{array}\right)_{n}, \quad j=h, v, \quad \text { (A3) }
\end{aligned}
$$

in which $\Phi_{j}=2 \pi Q_{j}\left(1-\eta_{e c}\right)$ is the betatron phase shift of the ions from the exit to entrance of electron cooler, $\eta_{e c}$ is the fraction of the cooling section length to the ring circumference, $\alpha_{j}, \beta_{j}$ and $\gamma_{j}$ are the Twiss parameters at the entrance. The kick strength calculated from the electron cooling $k_{j \text { cool }}$ and the IBS effect $k_{j \text { IBS }}$ [22] are

$$
\begin{aligned}
& k_{j \mathrm{cool}}=e^{\lambda\left(\vec{V}_{j}\right)\left(v_{i}-v_{e}\right) d t}, \\
& k_{j \mathrm{IBS}}=\frac{\xi}{\tau_{j}} .
\end{aligned}
$$

In the simulation, the horizontal and vertical betatron oscillations are assumed to be fully uncoupled.

\section{Synchrotron motion}

The synchrotron motion of the particles is affected by the combination of $\mathrm{rf}$ field, electron cooling, IBS and space-charge effect. In the code, the synchrotron motion is described by the symplectic mapping equation,

$$
\begin{gathered}
\Delta v_{n+1}=\Delta v_{n}+\Delta_{r f}+\Delta_{\text {cool }}+\Delta_{\mathrm{SC}}+\Delta_{\mathrm{IBS}}, \\
\phi_{n+1}=\phi_{n}+2 \pi \eta \frac{\Delta v_{n+1}}{v_{0}},
\end{gathered}
$$

where $\Delta v$ is relative velocity between the inspected particle and the synchronous particle, $\phi$ is the particle phase coordinate with respect to the ring instead of to the rf.

The change of particle velocity caused by rf and spacecharge effect and IBS are

$$
\begin{aligned}
\Delta_{r f} & =\frac{Z e V_{r f}}{A \beta^{2} E} v_{0}\left[\sin \left(h \phi_{n}\right)-\sin \phi_{s}\right], \\
\Delta_{\mathrm{SC}} & =\frac{Z e V_{S C}}{A \beta^{2} E} v_{0}, \\
\Delta_{\mathrm{IBS}} & =\frac{\xi}{\tau_{p}},
\end{aligned}
$$

where $V_{\mathrm{SC}}$ is the space-charge potential compared with the external rf potential.

The velocity change caused by the electron cooling $\Delta_{\text {cool }}=\vec{V}_{k+1}-\vec{V}_{k}$ can be calculated by

$$
\vec{V}_{k+1}=\left(\vec{V}_{k}-\vec{V}_{e}\right) e^{\lambda\left(V-V_{e}\right) d t}+\vec{V}_{e}
$$

where $\lambda$ is the cooling rate.

\section{APPENDIX B: ELECTRON COOLING}

The electron cooling rate can be simply calculated by

$$
\lambda(\vec{V})=\frac{\vec{F}}{M V_{i}} .
$$

The friction force $F$ on the particle moving with velocity $V_{i}$ with electron beam density $n_{e}$ is calculated by the Parkhomchuk cooling force in the particle reference frame,

$\vec{F}=-4 n_{e} m_{e} Z^{2} r_{e}^{2} c^{4} \frac{\vec{V}_{i}}{\left(V_{i}^{2}+V_{\mathrm{eff}}^{2}\right)^{3 / 2}} \ln \left(\frac{\rho_{\max }+\rho_{\min }+\rho_{L}}{\rho_{\min }+\rho_{L}}\right)$.

The impact parameters can be written as

$$
\begin{aligned}
\rho_{L} & =\frac{m_{e} V_{e \perp}}{e B}, \\
\rho_{\min } & =\frac{Z r_{e} c^{2}}{V_{i}^{2}}, \quad r_{e}=\frac{e^{2}}{m c^{2}}, \\
\rho_{\max } & =\frac{\sqrt{V_{i}^{2}+V_{\mathrm{eff}}^{2}}}{\omega_{e}+1 / \tau}, \quad \omega_{e}=c \sqrt{4 \pi n_{e} r_{e}},
\end{aligned}
$$

where $\omega_{e}$ is the plasma frequency of the electron beam, $\tau$ is the time of flight in the cooling section, $B$ is the longitudinal magnet field and $V_{e \perp}$ is the velocity of thermal motion electrons at the cooling section. The effective velocity of the center Larmor cycles $V_{\text {eff }}$ is described by

$$
V_{\mathrm{eff}}=\beta \gamma c \Delta B_{r m s},
$$

where $\Delta B_{r m s}$ is the deflection of magnetic field in the longitudinal at the cooling section.

\section{APPENDIX C: INTRABEAM SCATTERING}

In the simulation code, the Martini model is used to calculate the IBS heating rate,

$$
\begin{aligned}
\frac{1}{\tau_{p}} & =\left\langle\frac{n A}{2}\left(1-d^{2}\right) f_{1}\right\rangle, \\
\frac{1}{\tau_{x^{\prime}}} & =\left\langle\frac{A}{2}\left[f_{2}+\left(d^{2}+\tilde{d}^{2}\right) f_{1}\right]\right\rangle,
\end{aligned}
$$




$$
\frac{1}{\tau_{z^{\prime}}}=\left\langle\frac{A}{2} f_{3}\right\rangle
$$

where $n=1$ for bunched beam and $n=2$ for coasting beam. The angular brackets mean averaging over the lattice of CSRm:

$$
\begin{aligned}
A & =\frac{\sqrt{1+\alpha_{x}^{2}} \sqrt{1+\alpha_{z}^{2}} c r_{i}^{2} \lambda_{0}}{16 \pi \sqrt{\pi} \sigma_{x_{\beta}} \sigma_{x_{\beta}^{\prime}} \sigma_{z} \sigma_{z^{\prime}} \sigma_{p} \beta^{3} \gamma^{4}}, \\
\lambda_{0} & = \begin{cases}N / L & \text { for coasting beam } \\
N_{b} /\left(2 \sqrt{\pi} \sigma_{s}\right) & \text { for bunched beam. }\end{cases}
\end{aligned}
$$

The function $f_{i}$ is the integral of the beam parameters along the ring

$f_{i}=k_{i} \iiint \sin \mu g_{i}(\mu, \nu) \exp [-D(\mu, \nu) z] \ln \left(1+z^{2}\right) d \nu d \mu d z$,

with the coefficients $k_{1}=1 / c^{2}, k_{2}=a^{2} / c^{2}, k_{3}=b^{2} / c^{2}$, and

$$
\begin{gathered}
D=\frac{\left|\sin ^{2} \mu \cos ^{2} \nu+\sin ^{2} \mu(a \sin \nu-\tilde{d} \cos \nu)^{2}+b^{2} \cos ^{2} \mu\right|}{c^{2}}, \\
g_{1}(\mu, \nu)=1-3 \sin ^{2} \mu \cos ^{2} \nu, \\
g_{2}(\mu, \nu) 1-3 \sin ^{2} \mu \sin ^{2} \nu+6 \tilde{d} \sin \mu \sin \cos \nu / a, \\
g_{3}(\mu, \nu)=1-3 \cos ^{2} \mu .
\end{gathered}
$$

The normalized parameters $(a, b, c, d, \tilde{d})$ relate to the beam distribution and the lattice of ring; more details can be found in Ref. [22]. In the calculation, the growth rate on single model particle $\Delta \theta$ is multiplied by a standard Gaussian random number $\xi$,

$$
\Delta \theta=\lambda \xi
$$

[1] J. W. Xia et al., The heavy ion cooler-storage-ring project (HIRFL-CSR) at Lanzhou, Nucl. Instrum. Methods Phys. Res., Sect. A 488, 11 (2002).

[2] X. D. Yang, H. W. Zhao, J. W. Xia, W. L. Zhan, and B. W. Wei, HIRFL-CSR electron cooling devices, in Proceedings of the 2nd Asian Particle Accelerator Conference (APAC2001), Beijing, China (JACoW, Beijing, China, 2001), p. 777.

[3] A. V. Bubley, V. V. Parkhomchuk, and V. B. Reva, Advantages of electron cooling with radially varying electron beam density, Nucl. Instrum. Methods Phys. Res., Sect. A 532, 303 (2004).

[4] A. Bubley, A. Goncharov, A. Ivanov, E. Konstantinov, S. Konstantinov, A. Kryuchkov, V. Panasyuk, V. Parkhomchuk, V. Reva, B. Skarbo, B. Smirnov, B. Sukhina, M. Tiunov, M. Zakhvatkin, and X. Yang, The electron gun with variable beam profile for optimization of electron cooling, in Proceedings of the 8th European Particle Accelerator Conference (EPAC2002), Paris, France (JACoW, Paris, France, 2002), p. 1356.

[5] P. Li, Y. J. Yuan, J. C. Yang, J. W. Xia, W. Zhang, R. S. Mao, D. Y. Yin, and W. P. Chai, The status and improvement of the HIRFL-CSR operation software, Nucl. Instrum. Methods Phys. Res., Sect. A 697, 17 (2013).

[6] X. H. Cai, R. C. Lu, C. J. Shao, F. F. Ruan, D. Y. Yu, M. S. Li, W. L. Zhan, D. K. Torpokov, and D. Nikolenko, Test results of the HIRFL-CSR cluster target, Nucl. Instrum. Methods Phys. Res., Sect. A 555, 15 (2005).

[7] H. Poth, Electron cooling: Theory, experiment, application, Phys. Rep. 196, 135 (1990).

[8] K. Noda, S. Shibuya, D. Tann, S. Ninomiya, T. Uesugi, T. Furukawa, T. Honma, T. Iwashima, H. Y. Ogawa, Y. Hashimoto, T. Fujisawa, H. Uchiyama, T. Uesugi, M. Muramatsu, and E. Syresin, Electron cooling of bunched ion beam at NIRS-HIMAC, Nucl. Instrum. Methods Phys. Res., Sect. A 532, 129 (2004).

[9] K. A. Brown, Development of micro-bunching beams and application to rare K decay experiments, http://slideplayer .com/slide/4376943/.

[10] Concept for an electron ion collider (EIC) detector built around the BaBar solenoid, https://arxiv.org/pdf/1402 .1209.pdf.

[11] W. Z. Zhang, Z. Xu, X. D. Yang, H. W. Zhao, and W. L. Zhan, RF acceleration system at HIRFL-CSR main ring, in Proceedings of the 2003 Particle Accelerator Conference, Portland, Oregon (JACoW, Portland, Oregon, 2003), p. 1288.

[12] G. Kalisch, K. Beckert, B. Franzke, I. Hofmann, and U. Schaaf, Longitudinal space charge effects in cooled bunched beams, in Proceedings of the 3rd European Particle Accelerator Conference, Berlin, Germany (JACoW, Berlin, Germany, 1992), p. 780.

[13] T. J. P. Ellison, S. S. Nagaitsev, M. S. Ball, D. D. Caussyn, M. J. Ellison, and B. J. Hamilton, Attainment of SpaceCharge Dominated Beams in a Synchrotron, Phys. Rev. Lett. 70, 790 (1993).

[14] L. J. Mao, H. Zhao, X. D. Yang, J. Li, J. C. Yang, Y. J. Yuan, V. V. Parkhomchuk, V. B. Reva, X. M. Ma, T. L. Yan, M. T. Tang, and J. W. Xia, Longitudinal electron cooling experiments at HIRFL-CSRe, Nucl. Instrum. Methods Phys. Res., Sect. A 808, 29 (2016).

[15] V. V. Parkhomchuk, New insights in the theory of electron cooling, Nucl. Instrum. Methods Phys. Res., Sect. A 441, 9 (2000).

[16] Y. N. Rao and T. Katayama, Simulation of electron cooling and intrabeam scattering processes of a heavy ion beam in HIRFL-CSR, Nucl. Instrum. Methods Phys. Res., Sect. A 391, 42 (1997).

[17] M. Martini, Report No. CERN PS/84-9 (AA), Geneva, 1984, https://cds.cern.ch/record/151638/files/cm-p00047664.pdf. 
[18] C. R. Prior, Computer simulation of the motion of chargedparticle beams under space charge, RAL-TR-98-048, 1998, http://cds.cern.ch/record/360424/files/SCAN-9807100 .pdf.

[19] S. Nagaitsev, T. Ellison, M. Ellison, and D. Anderson, The investigation of space charge dominated cooled bunched beams in a synchrotron, in Workshop on Beam Cooling and Related Topics, Montreux, Swichzerland, 1993 (1994), CERN-1994-003, Vol. 94, p. 405, http://cds.cern.ch/ record/398779/files/p405.pdf.

[20] S. Y. Lee, Accelerator Physics (World Scientific, Shanghai, 2006), Vol. 2, p. 255.
[21] S. Nagaitsev, D. Broemmelsiek, A. Burov, K. Carlson, C. Gattuso, M. Hu, T. Kroc, L. Prost, S. Pruss, M. Sutherland, C. W. Schmidt, A. Shemyakin, V. Tupikov, A. Warner, G. Kazakevich, and S. Seletskiy, Experimental Demonstration of Relativistic Electron Cooling, Phys. Rev. Lett. 96, 044801 (2006).

[22] I. Meshkov, A. Sidorin, A. Smirnov, G. Trubnikov, and R. Pivin, BETACOOL Physics Guide, http://betacool.jinr.ru/ programs/.

[23] C. Walck, Handbook of statistical distributions for experimentalists, 2007, http://home.agh.edu.pl/ mariuszp/ wfiis_stat2/handbook_stat_distr.pdf. 